Musées, Patrimoine et Culture scientifiques et techniques

$123 \mid 2009$

mai - juin 2009

\title{
Le patrimoine géologique en prise avec son territoire
}

\section{Myette Guiomar}

\section{OpenEdition \\ Journals}

Édition électronique

URL : http://journals.openedition.org/ocim/238

DOI : $10.4000 /$ ocim.238

ISSN : 2108-646X

Éditeur

OCIM

Édition imprimée

Date de publication : 1 mai 2009

Pagination : $31-39$

ISSN : 0994-1908

Référence électronique

Myette Guiomar, « Le patrimoine géologique en prise avec son territoire », La Lettre de I'OCIM [En ligne], 123 | 2009, mis en ligne le 01 mai 2011, consulté le 02 mai 2019. URL : http:// journals.openedition.org/ocim/238; DOI : 10.4000/ocim.238 


\section{La Réserve Naturelle géologique de Haute-Provence}

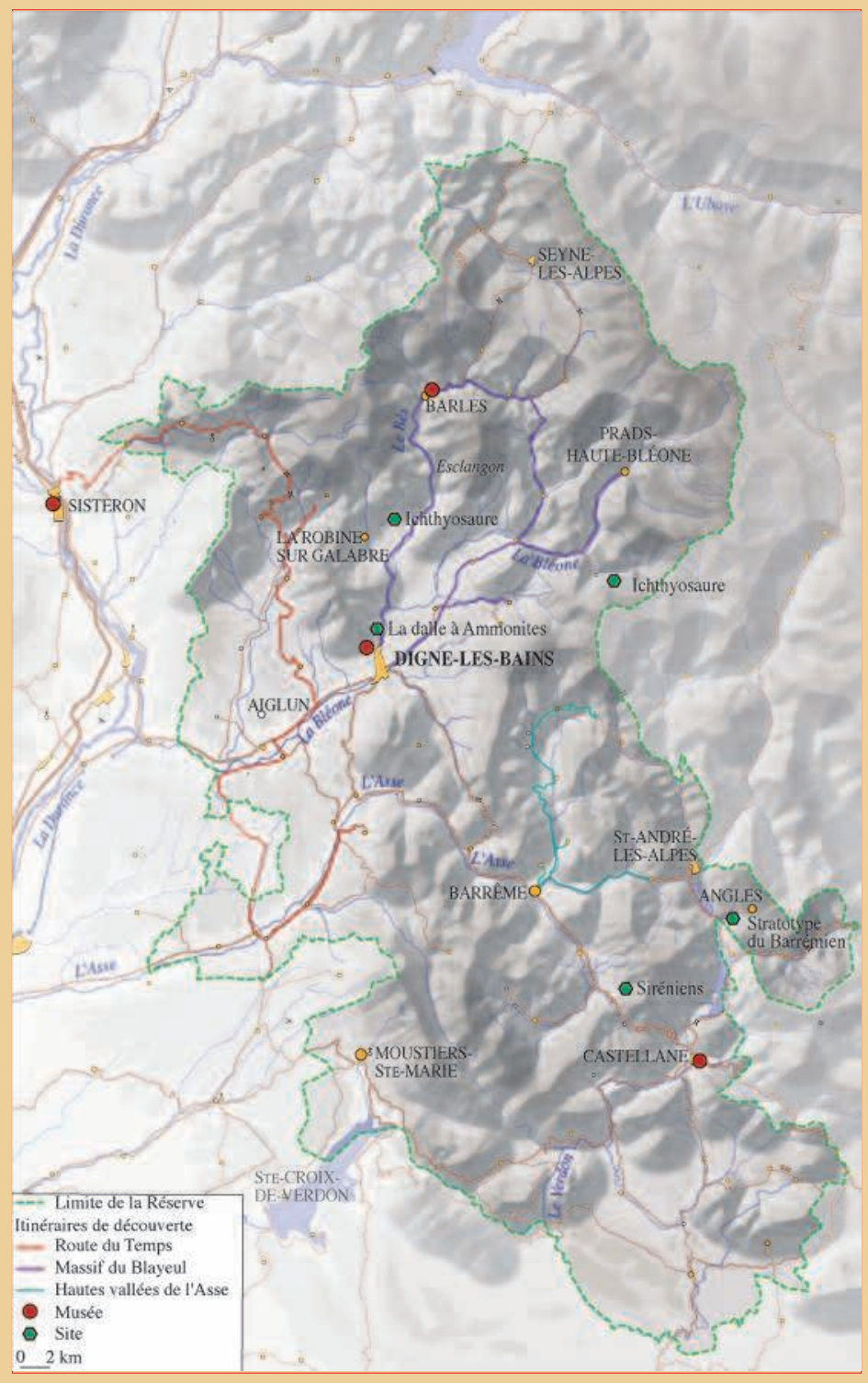

(c) RGHP
La Réserve Naturelle géologique de Haute-Provence est une Réserve Naturelle Nationale, créée en 1984 ; elle fait donc partie du réseau « Réserves Naturelles de France » (www.reserves-naturelles.org). Comme onze autres RNN, sa création a été motivée par la protection du patrimoine géologique. Gérée par une association loi 1901, elle est soutenue par l'État, le Conseil régional Provence-Alpes-Côte-d'Azur, le Conseil général des Alpes de Haute-Provence, la Ville de Digneles-Bains.

Dix-huit sites représentant 270 ha sont classés en réserve naturelle nationale ; ils bénéficient à ce titre d'une protection juridique « rapprochée ». Parmi ces sites, certains ont été valorisés pour le public comme par exemple la dalle aux ammonites de Digne, l'ichtyosaure de La Robine, ou le récif fossile de Saint-Lions. Un périmètre de protection a été créé à partir de 1989 par arrêté préfectoral ; une réglementation moins contraignante que sur les sites classés permet de préserver le patrimoine géologique de cette zone tout en développant des actions de valorisation. Ce périmètre s'est agrandi au fil des ans à la demande d'adhésion de nombreuses communes ; il représente aujourd'hui 230000 ha avec 52 communes des Alpes de HauteProvence et 7 du Var.

Plus grande réserve géologique d'Europe, elle est également membre fondateur des European Geoparks (www.europeangeoparks.org); ces Geoparks ont pour vocation de développer le géotourisme sur leurs territoires et à travers l'Europe. Aujourd'hui la Réserve géologique de Haute-Provence est un territoire labellisé par les politiques de l'UNESCO et reconnue membre du réseau des UNESCO global geopark network.

Réserve Naturelle géologique de Haute-Provence
Montée Bernard Dellacasagrande
BP 156
04005 Digne-les-Bains cedex
téléphone + 33492367070
contact@resgeol04.org
www.resgeol04.org


proches des oursins, que les orfèvres de Digne ont eu l'idée de monter en bijoux. À la fin du XIXe siècle, les Bas-Alpins partis au Mexique pour tenter de faire fortune portaient ces bijoux en signe de reconnaissance. La production des bijoux s'est poursuivie jusque dans les années 1960 puis a périclité. Les ammonites, mollusques marins à coquille spiralée, et qui ont vécu à l'époque des dinosaures, sont des fossiles que l'on trouve en abondance en Haute Provence ; près de Digne, en bord de route, une couche calcaire était couverte de ces gros « escargots pétrifiés ». La dalle aux ammonites au milieu du XXe siècle n'était pas encore devenue le site emblématique de Digne ; cela dit les Dignois semblaient déjà attachés à ces pierres et enclins à les protéger sur les quelques mètres carrés qui affleuraient alors. Ainsi un grillage avait été posé pour empêcher les visiteurs et collectionneurs de fossiles d'exploiter le gisement.

L'arrivée à Digne en 1978 d'un jeune étudiant - Guy Martini - envoyé par l'université de Provence (à la demande de la Ville) pour réaliser un inventaire des sites géologiques des environs, allait changer la donne. Soutenu par un groupe d'élus locaux et d'universitaires, Guy Martini a pu faire émerger l'idée d'une Réserve naturelle, malgré l'opposition farouche d'une partie de la population (peur de voir arriver des gardes) et de certains universitaires. Ces derniers craignaient d'une part de n'avoir plus accès à certains sites mis «sous cloche » et redoutaient d'autre part que la publicité faite autour de la Réserve, accroisse dangereusement l'exploitation des sites fossilifères de la région par les collectionneurs et surtout par les marchands de fossiles. La Réserve géologique « des environs de Digne » a tout de même vu le jour officiellement après 5 années de négociations à toutes les échelles. Entre temps, un premier chantier de fouilles mené sur la dalle de Digne, a permis de mettre à l'air libre plus de 500 ammonites sur une surface de $160 \mathrm{~m}^{2}$. Le site resté libre d'accès n'a pas fait depuis l'objet de tentative de pillage. Le décret ministériel créant la Réserve (1984) puis l'arrêté préfectoral définissant le périmètre de protection (1989) ont offert un cadre réglementaire visant à enrayer le pillage des sites. Un garde a désormais été affecté à la surveillance des zones sensibles. La répression n'ayant jamais été le meilleur outil pour assurer la protection des gisements, la Réserve a développé depuis ses origines, une politique de sensibilisation à destination de la population locale (dont certains collectionneurs locaux) et surtout des enfants. Des milliers d'enfants ont profité des classes de découverte organisées par la Réserve ; avec le recul des années et grâce au témoignage de ceux qui ont parcouru en culottes courtes la «Trace des Anciens » ou les « Sentiers du Verdon » il est possible de mesurer l'impact que ce type d'expérience a eu sur les consciences. Les enfants qui ont vécu ces aventures ont compris que les roches et les fossiles transmettaient un message du passé ; ils ont appris à les apprécier et à les respecter. Devenus adultes, ils sont prêts aujourd'hui à intervenir pour la défense et le respect du patrimoine géologique.

La Ville de Digne a mis à disposition de la Réserve une propriété, avec une bâtisse ancienne réhabilitée en centre de géologie (depuis devenu le musée Promenade). Le centre, avec ses salles d'expositions (fossiles, aquariums d'eau de mer, art et science...) et son parc, a évolué en un lieu de rencontres et d'échanges. Toutes sortes de manifestations y ont été organisées de façon à inviter des publics très variés à venir à la rencontre du patrimoine géologique. Des sites géologiques comme la dalle aux ammonites ou l'ichtyosaure (reptile marin du Secondaire) de La Robine, ont été aménagés pour être librement visités par le public.

L'implication d'une poignée de personnes (dont Guy Martini à l'échelle nationale puis internationale) pour faire reconnaître le patrimoine géologique a permis de faire émerger cette notion, de faire naître les premières réserves géologiques et d'en faire la promotion. L'organisation à Digne du premier symposium international sur le patrimoine géologique a donné à la Réserve un rayonnement international. L’impact de cet événement est important y compris localement; ce n'est en effet pas un hasard si dans les années qui ont suivi, de nombreuses communes ont demandé leur adhésion à la Réserve.

Le regard porté sur cet espace naturel par les élus et par leurs administrés a alors bien changé : on n’a plus vu dans la Réserve une menace (mise sous cloche, répression, gardes, surveillance...) mais un outil de valorisation et de promotion d'un territoire, avec de possibles retombées économiques induites. Victime de son succès, la Réserve géologique n’a pu répondre favorablement à toutes les demandes; elle compte toutefois aujourd'hui 59 communes, contre 36 en 1991.

\section{De la sensibilisation au développement}

L'après 1991 est une phase de développement avec l'extension du territoire, l'accroissement de l'image internationale de la Réserve (le moulage de la Dalle aux ammonites pour le Japon...), le début d'un mariage réussi entre la Réserve géologique et l'art 


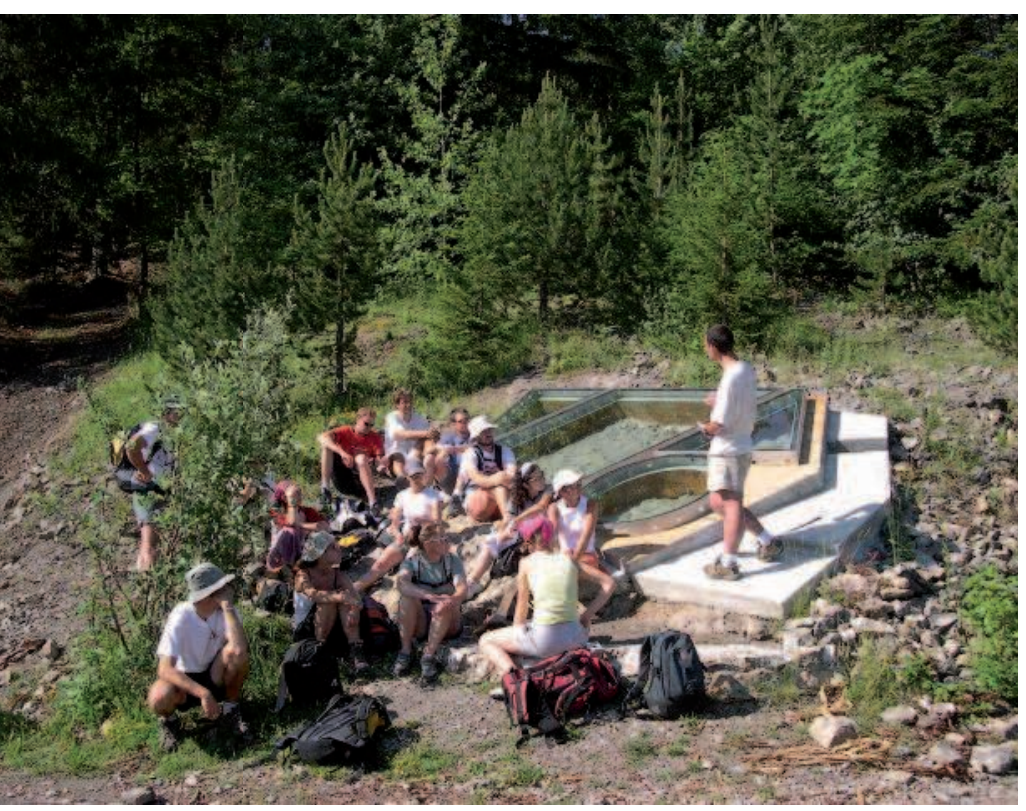

La découverte géologique avec un accompagnateur en montagne : ici les ammonites de Fontbelle sur « La Route du Temps » (c) RGHP

contemporain, et les programmes de développement durable autour de patrimoine géologique. Seuls les deux derniers aspects seront abordés ici.

Au cours des années 1990 sont lancés les programmes européens Leader II qui avaient pour objectif de soutenir en zones rurales défavorisées des projets innovants de développement local. Dans ce contexte, la Réserve géologique a élargi son champ d'action vers l'économie locale. Le projet construit autour d'une réserve géologique re-pensée comme une "entreprise-territoire ", a été soutenu par les institutions et les élus. Le pays dignois n'existait pas encore et le territoire avait besoin de tels projets d'envergure pour se structurer. La RGHP a conçu un projet de développement touristique autour du patrimoine géologique impliquant de très nombreux partenaires locaux. Une politique de réseau a été développée. Afin d'impliquer la population locale et les différents acteurs, des rencontres et des formations ont été organisées par la réserve. Pour que le tissu local adhère au projet et se l'approprie, il était nécessaire de faire (encore plus) évoluer le regard porté sur le patrimoine géologique. Des circuits de découverte ont été conçus de façon à accroître la fréquentation touristique dans les zones défavorisées ; les accompagnateurs en montagne ont été formés à la géologie, leur permettant ainsi de proposer de nouvelles activités à destination du grand public et des scolaires; de nouveaux produits inspirés de la géologie ont été créés par des artisans.
Ainsi les pains en forme d'ammonites ont connu un vif succès. La géologie et en particulier les fossiles ont changé de statut; le tas de cailloux est devenu synonyme de patrimoine dont on peut espérer notoriété et retombées économiques.

Avec l'aménagement de circuits de découverte tels que « La Route du Temps », « Les hautes vallées de l'Asse $» .$. et la réalisation d'antennes muséographiques situées aux portes de la Réserve comme le musée Sirènes et Fossiles à Castellane, le musée Terre et Temps à Sisteron, la maison de la Géologie à Barles, c'est l'ensemble du patrimoine local qui visait à être valorisé et non pas le seul patrimoine géologique. Ainsi chaque circuit a été accompagné de la publication d'un ouvrage réunissant tout ce qui fait la richesse et la diversité d'une de ces petites unités géographiques : histoire locale, économie, faune, flore, architecture. Le succès de ces guides tient principalement dans le fait que les habitants y retrouvent ce qui fait que «leur pays est leur pays ", avec des hommes et des femmes qui l'ont construit, modelé, des gens qui l'habitent aujourd'hui et le font vivre ; ce pays n’appartient pas au passé géologique ou historique c'est un pays où chacun construit son avenir.

\section{Art et science ? L'adhésion du public}

Au cours de la même période se sont développés les projets d'art contemporain. Cette aventure a réellement commencé lors de la célébration des 10 ans de la Réserve. Sur toute l'année 1994, la RGHP en collaboration avec le musée de Digne a sollicité des artistes pour venir créer, s'exprimer sur le territoire et surtout dans le musée de la Réserve à Digne. L'idée essentielle était de mélanger les genres, en invitant des publics variés à se rencontrer, à échanger et autour d'un événement de les amener à percevoir ce que cette notion de patrimoine géologique pouvait recouvrir. Cette année a vu son apothéose avec $L a$ Nuit de la mémoire de la Terre. Un grand spectacle déambulatoire conçu spécialement par la compagnie " Générik vapeur », a été le clou de cette nuit où se sont multipliées dans le parc et dans le musée, toutes sortes de représentations et activités (danse, thêâtre, chant, céramique, cuisine néolithique...). Cet événement a eu l'intérêt de toucher un public jusque-là réfractaire à tout ce qui peut évoquer la géologie ; sur les 3000 personnes venues assister et participer à la fête, de nombreux Dignois ont reconnu avoir découvert la Réserve géologique à cette occasion, certains sont désormais des fidèles. Alechinsky a réalisé la nouvelle affiche de la Réserve dans le cadre de cet anniversaire; de nombreux artistes de 


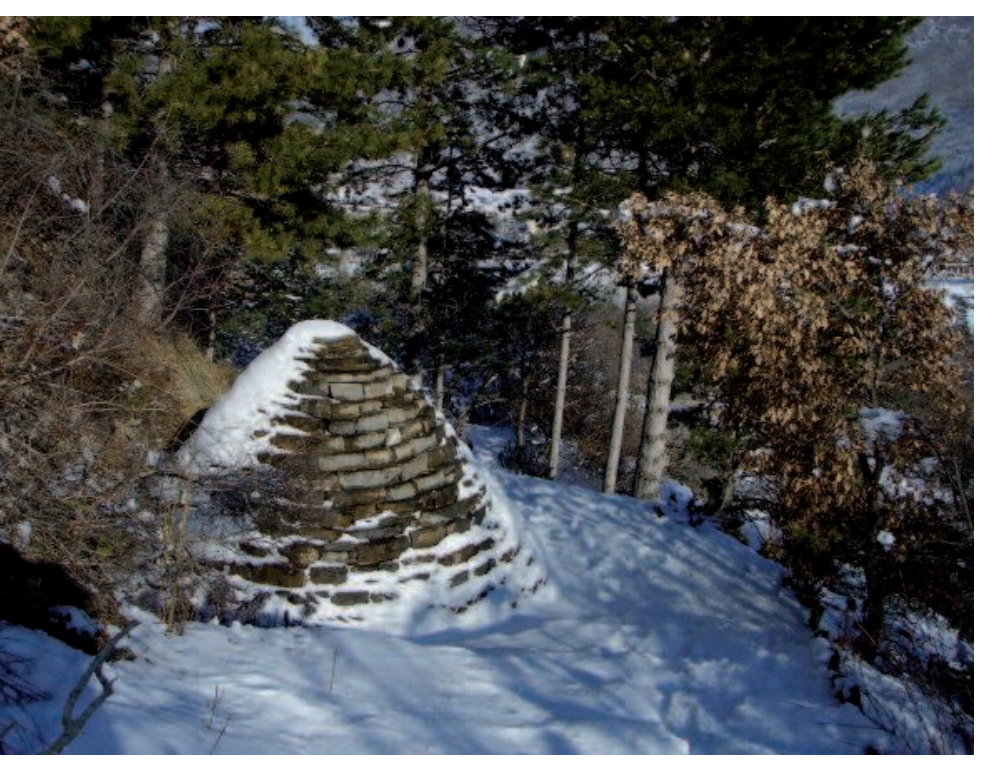

Le sentier des cairns au musée Promenade : une des premières œuvres pérennes d'Andy Goldsworthy sur le territoire de la Réserve.

(c) RGHP

renom se sont peu à peu approprié le patrimoine géologique et surtout ont été séduits par une expérience nouvelle autour de la géologie et par l'ambiance particulière qui se dégage des paysages de la Haute-Provence. C'est également au cours de l'année 1994, qu'Andy Goldsworthy, artiste de renommée mondiale, invité par la Réserve, a réalisé ses premières œuvres éphémères sur le territoire.

Quelques années plus tard, la collaboration entre la Réserve géologique et le musée Gassendi de Digne, a donné naissance au Cairn : Centre d'Art Informel de Recherche sur le Nature. Dans ce cadre, un nouveau projet avec Andy Goldsworthy a vu le jour : « Refuges d'Art ». Un sentier de randonnée pédestre partant du musée Gassendi à Digne parcourt une grande boucle de 134 kilomètres à travers les massifs de la Réserve. L'itinéraire est ponctué de «Refuges », sites choisis par l'artiste. Ainsi, chapelles, abris de bergers, maison des Résistants, tous ces lieux ruinés ont été reconstruits à l'identique, lorsque c'était possible, et conçus de façon à héberger de façon pérenne une création et accueillir les randonneurs, le temps d'une pose ou pour une nuit. L'œuvre contemporaine et le bâti « ancien » sont indissociables et font partie intégrante d'une seule et même création de l'artiste. Ce travail de réhabilitation de sites à l'abandon a contribué à véhiculer une nouvelle image de la Réserve géologique et de ses actions. Grâce aux projets d'art contemporain en lien direct avec le patrimoine géologique, la population locale a vu renaître une partie de son histoire qui sombrait dans l'oubli. Le fait qu'un artiste majeur soit tombé amoureux de la Réserve géologique et qu'il ait choisi leur petit patrimoine local pour donner vie à un grand projet a généré un sentiment profond de respect.

À la suite de quelques rencontres avec l'artiste, mais surtout grâce au bouche à oreille, le projet a séduit les élus, les habitants des communes concernées par le projet mais aussi des autres communes du territoire qui espèrent des développements avec de nouveaux « Refuges ».

\section{Une implication et des initiatives locales}

La Réserve géologique récolte à présent les fruits de ce qu'elle a semé au cours de longues années d'existence. Cependant la Réserve prend aussi la mesure des limites de certaines expériences qu'elle a menées notamment dans le cadre des programmes européens. Lorsque les programmes sont terminés, l'absence de moyens pour poursuivre à plus long terme les actions mises en place se fait cruellement sentir. Les aménagements vieillissent, faute de financement pour remplacer le mobilier, les réseaux de partenaires ne fonctionnent plus faute de personnel dédié à cet effet. Les réalisations de la Réserve ont néanmoins marqué les esprits comme décrit plus haut et la population locale qui a globalement adhéré aux projets de la Réserve prend désormais le relais. Ainsi la RGHP ne pouvant plus assurer la gestion des antennes muséographiques réalisées dans le cadre de Leader II, ce sont les villes de Sisteron et de Castellane et le SIVOM de Seyne-les-Alpes, qui l'ont reprise à leur charge, la Réserve demeurant leur partenaire pour le suivi des expositions. Sans cette réappropriation du patrimoine géologique par les élus et les habitants, ces musées étaient voués à la fermeture. Sur chaque site, de nouvelles initiatives ont vu le jour : les lieux ont été enrichis. Le musée des Sirènes et des Fossiles de Castellane héberge désormais d'autres partenaires tels que le Parc Naturel Régional du Verdon et l'association Petra castellana qui propose des expositions sur l'histoire locale.

D'autres initiatives voient régulièrement le jour sur le territoire de la RGHP, portées par les communes et les pays. La Réserve géologique est alors sollicitée en tant que partenaire scientifique et en tant qu'expert en patrimoine géologique. La commune d'Angles près de Saint-André-les-Alpes a ainsi sollicité la RGHP pour l'aménagement d'un de ses sites : le fameux stratotype du Barrémien (1). La RGHP n'avait rien programmé pour cette coupe géologique, en tout cas 


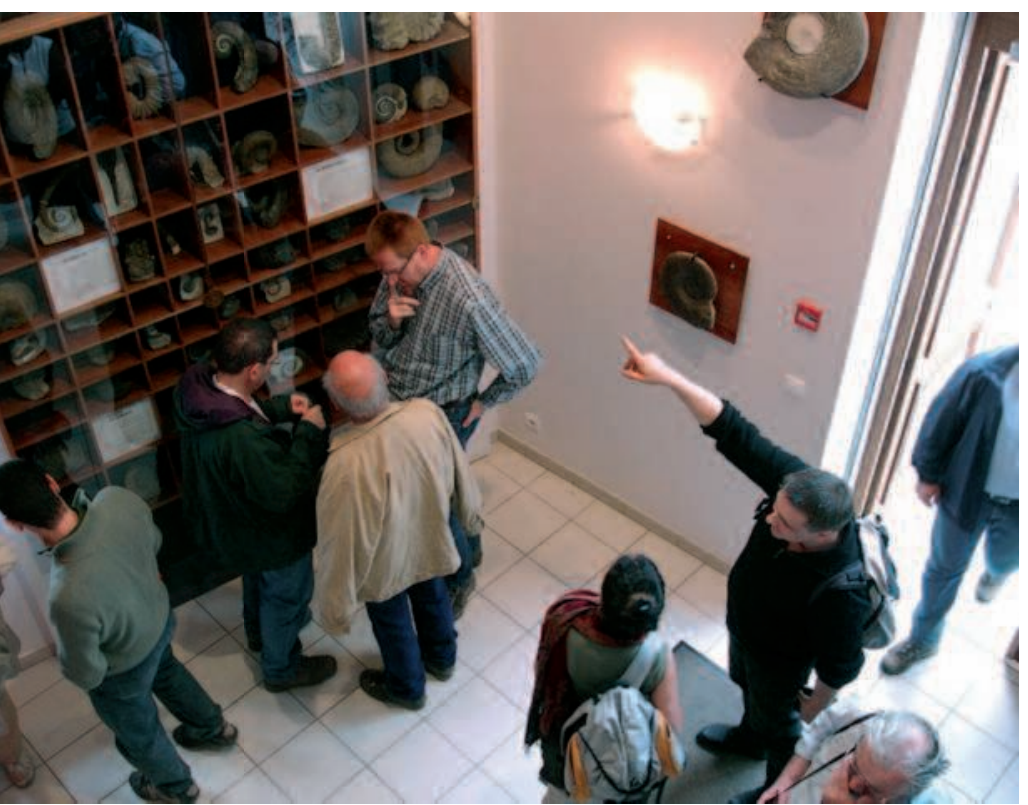

Les congressistes de l'association Paléontologique française découvrent la collection Maurel dans le hall de la mairie de Barrême.

(c) RGHP

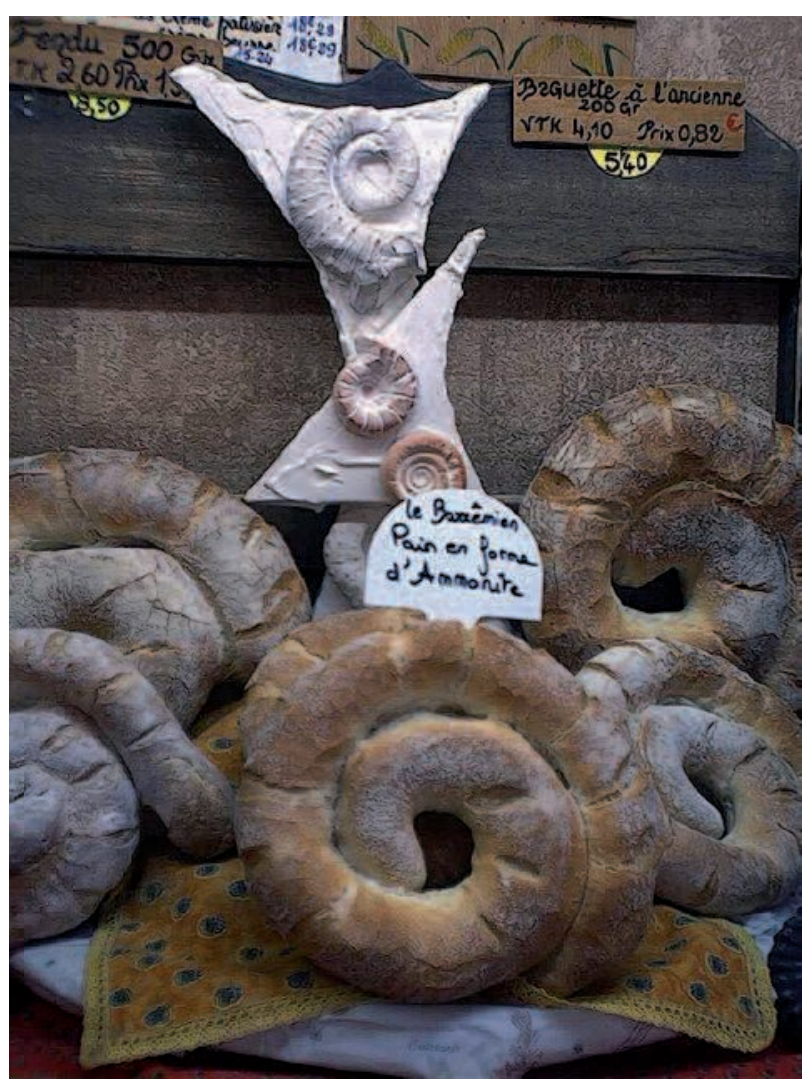

La vitrine d'une boulangerie de Barrême avec des pains en forme d'ammonites et d'étoiles de Saint-Vincent (c) RGHP pas dans un avenir proche. Le projet a été porté par la commune grâce aux contrats de Pays avec le soutien de l'Europe (Leader +). La commune d'Aiglun (Pays Dignois) a elle aussi commandé à la Réserve un aménagement de sentier géologique, venant enrichir et compléter le circuit de « La Route du Temps ».

La commune de Barrême, lieu emblématique du patrimoine géologique et localité éponyme du Barrémien, a ainsi développé l'image " géologique » au cœur même du village. Les ammonites sont présentes partout, sous forme de moulages (RGHP) dans les rues, au fond du bassin, sur le pavage à l'entrée de la mairie, ou sous forme d'originaux avec la collection Maurel. La plus belle réussite de la commune est celle de la valorisation de cette collection de fossiles au sein de la maison publique. Il y a quelques années, Marthe Maurel a légué à la commune l'exceptionnelle collection de fossiles qu'ellemême et son mari Louis Maurel avaient passionnément et patiemment constituée tout au long de leur vie. Louis et Marthe étaient de véritables figures locales, appréciées tant des habitants de Barrême que des nombreux collectionneurs et géologues venus parfois de loin pour visiter leur «musée ».

Cette collection fait partie intégrante de l'histoire et du patrimoine local ; le maire de l'époque Jean-Marie Gibelin a conçu l'idée originale d'accueillir la collection au sein même de la mairie. Le projet a été confié à l'architecte et muséographe Éric Klein, et à l'artiste peintre Georges Autard. Les fossiles ont ainsi été mis en scène dans l'espace du hall d'entrée ainsi que dans la salle du conseil. Ici encore le rôle particulier de l'artiste est à souligner : Georges Autard avait en effet conçu pour le musée Promenade la première exposition mariant l'art, l'histoire des sciences et l'esthétique des ammonites dites déroulées (et pour l'essentiel datées du Barrémien). Cette création faisant naître l'émotion participe à la sensibilisation du public. L'approche esthétique et historique du fossile lui donne de la valeur tout en le rendant plus accessible au public non averti. Lartiste qui avait si bien mis en scène les ammonites barrémiennes au musée Promenade était tout indiqué pour la nouvelle exposition de Barrême. Les acteurs locaux se sont donc non seulement appropriés le patrimoine géologique, mais aussi la création contemporaine associée. L'artiste « récidiviste » s'est donc lui aussi approprié ce patrimoine minéral pour lui donner une seconde vie à Barrême. 


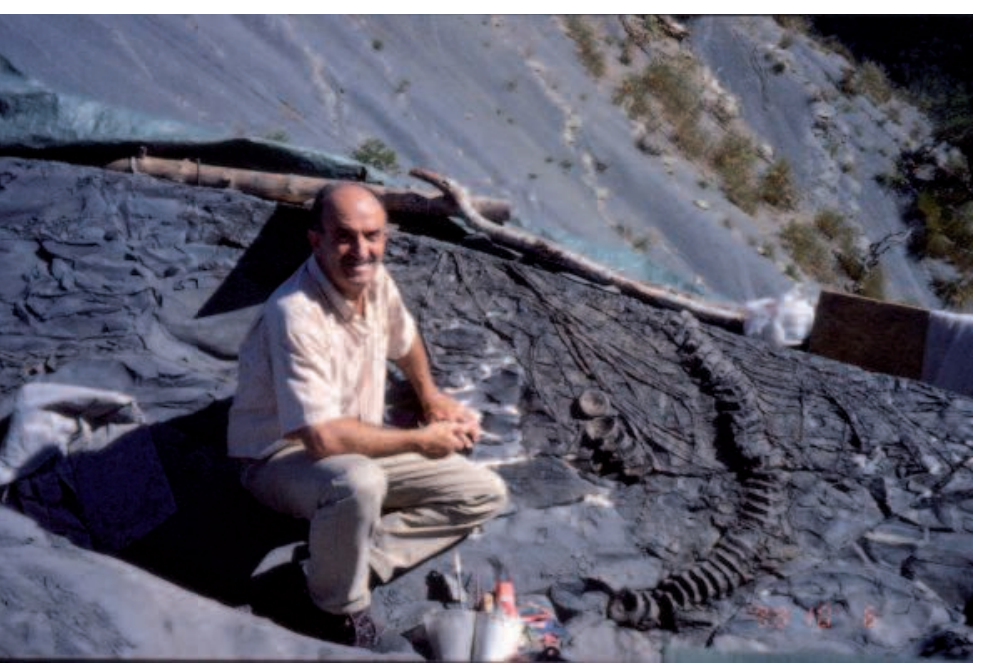

Le squelette de I'Ichtyosaure et Bernard Bartolini, maire de Prads-Haute-Bléone, 2003

(c) RGHP

Prads-Haute-Bléone est une commune de montagne qui est en passe de devenir célèbre grâce à ses ichtyosaures ; dans le village personne n'ignore qu'il s'agit de reptiles marins qui vivaient au temps des dinosaures. Les découvertes de ces fossiles et en particulier du dernier spécimen avait conduient la Réserve à élaborer un simple projet scientifique. Le chantier de fouilles ne devait pas être suivi d'une valorisation sur place du squelette, le site ne se prêtant pas vraiment à ce type de projet. Pourtant aujourd'hui le fossile est abrité dans une petite structure vitrée construite en bardeaux de mélèze, sur le lieu même de la découverte, dans un cadre aérien. L'ichtyosaure de la Mélaie est visité en été par de nombreux randonneurs. On en parle dans des colloques spécialisés. Que s'est-il passé ? Tout simplement la découverte ne justifiait pas l'extraction du fossile pour une étude plus approfondie en laboratoire, et surtout la population locale a souhaité conserver «son ichtyosaure » sur place. La gestion des sites doit être décidée d'un commun accord entre les gestionnaires du patrimoine géologique, les propriétaires des terrains, les élus et la population locale. Dans le cas présent, le site étant communal, l'autorisation de fouille a été demandée à Bernard Bartolini, maire de Prads-Haute-Bléone, et au final après une rencontre au sommet avec divers représentants de la commune, de la Réserve et de l'université de Provence, la décision a été prise de valoriser in situ le fossile. Le chantier, qui avait duré plus d'un mois durant l'été 2003, n'est pas passé inaperçu. La simple visite de quelques curieux locaux venus presque timidement voir ce que la géologue faisait là-haut, a facilement laissé la place à une implication totale : il était dit que les habitants de Chanolles (le hameau voisin) devaient participer au dégagement de l'ichtyosaure chanollais ! La première saison de fouille n'était pas encore terminée, et le projet pas encore élaboré, que quelques hardis volontaires attaquaient à coup de pioches les pentes de la montagne là où l'ancien chemin avait disparu. Aujourd'hui la Réserve assure le suivi technique de ce site considéré comme un site expérimental. La commune quant à elle a pris en charge l'entretien du sentier d'accès, qui demande plusieurs interventions annuelles compte tenu de son exposition. Cette expérience a récemment été présentée dans le cadre des « 4e Journées nationales du Patrimoine géologique », qui se sont tenues à Digne en septembre 2008 : poussant jusqu'au bout l'expérience de la collaboration entre la Réserve, la commune et l'université, le maire devenu congressiste et co-auteur de la communication consacrée à l'étude et la valorisation de «son ichtyosaure » a été abondamment questionné sur son implication. Les actes du colloque seront publiés fin 2009 dans la revue en ligne du BRGM (http://geolfrance.brgm.fr); les résumés des communications sont disponibles sur le site de la Réserve géologique (www.resgeol04.org/4es-journees.html).

\section{Les limites d'une expérience}

Des élus et des techniciens, de divers territoires de France ou de l'étranger, viennent de plus en plus nombreux découvrir ce qui a été réalisé en Haute-Provence. Ils souhaitent utiliser à leur tour le patrimoine

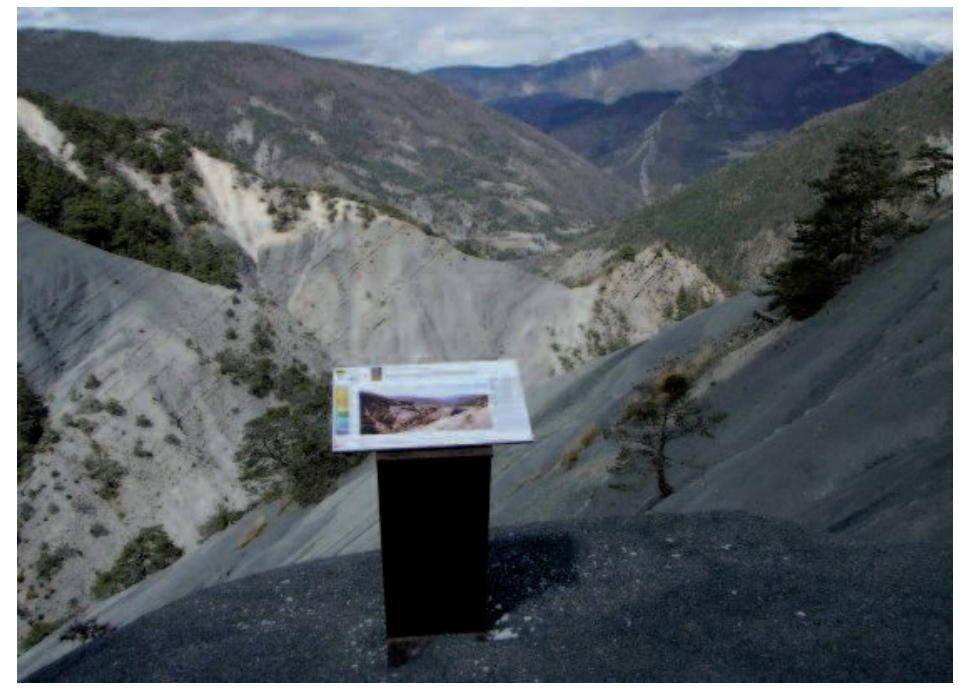

Une tablette de lecture pour découvrir l'environnement géologique de l'Ichtyosaure de la Mélaie (c) RGHP 


\section{Pour conclure}

Ce tour d'horizon des actions menées pour et avec les populations locales n'est bien évidemment pas exhaustif ; ces expériences peuvent sembler déjà à la fois nombreuses et variées ; elles ne sont cependant pas encore suffisantes, pour un territoire aussi étendu que celui de la Réserve. La RGHP doit donc encore aller de l'avant, expérimenter de nouvelles voies et travailler plus étroitement encore avec les acteurs locaux du développement (les Pays Dignois, Asse-Vaïre-Var-Verdon, Sisteronnais-Buech, le Parc Naturel Régional du Verdon...).

La célébration de l'Année internationale de la Planète Terre (2007, 2008 et 2009), a été pour la Réserve l'occasion de développer des projets phares impliquant de nombreux partenaires locaux, régionaux, nationaux et internationaux. Des « $4 \mathrm{e}$ journées nationales du patrimoine géologique », à l'opération «Enfants de la Terre » et en passant par la Fête de la Terre, toutes sortes de publics se sont investies, ont été sensibilisées ou se sont mobilisées pour ce patrimoine. L'action menée avec les enfants se poursuivra quant à elle jusqu'à la réalisation du « Monument aux Enfants de la Terre », et au-delà : la mémoire de la Terre se conjugue au passé au présent et au futur.

De nouvelles actions sont à imaginer pour impliquer toujours plus de monde pour le patrimoine géologique. La Réserve Naturelle géologique de HauteProvence a atteint l'âge mûr ; les enfants nés sur le territoire après sa création (1984), éduqués dans le respect du patrimoine géologique, et devenus de jeunes adultes ne pourraient-ils pas, dans cette optique, devenir une nouvelle force de proposition ?

\section{Note}

(1) Un stratotype est une coupe géologique (succession de couches) qui sert d'étalon à l'échelle internationale pour décrire un étage géologique. Le nom d'un étage est souvent lié à la localité qui a servi à sa description. Ainsi l'étage du Barrémien (130 à 135 millions d'années, Crétacé) a été décrit à partir d'observations faites dans les environs de Barrême par Coquand en 1862. Une centaine d'années plus tard, le stratotype du Barrémien a finalement été défini sur la commune d'Angles, à une vingtaine de kilomètres de la localité éponyme.

\section{Bibliographie}

De Wever, P. Un inventaire du patrimoine géologique pour la France, la Lettre de l'OCIM, n¹21, 2009, pp. 12-18.

Goldsworthy, A., Gomez, N., Brovelli, J.-P., Guiomar, M. et Magnaudeix, I. Refuges d'Art-Andy Goldsworthy. Fage édition, 2008, 224 p., 1 DVD

Guiomar, M. et Pagès, J.-S. Développement durable, tourisme et patrimoine géologique : l'exemple de la Réserve Naturelle géologique de Haute-Provence, BRGM, Geoscience e-Journals, à paraître.

Martini, G. (dir.) Actes du premier symposium international sur la protection du patrimoine géologique, Digne-les-Bains, 11-16 juin 1991, Mémoires de la Société Géologique de France, n¹65, 1994, 276 p.

Vésian, H. Les Hautes Vallées de l'Asse. Les Petites Affiches, 1999, 254 p.

Vésian, H. La Route du Temps. Les Petites Affiches, 2003, 351 p.

Vésian, H. La Route du Temps. Les Petites Affiches, 2006, 307 p. 